\title{
Análisis de la relación entre actividad empresarial y desigualdad económica *
}

\author{
Analysis of the Relationship between Business Activity and Economic Inequality
}

\author{
Juan Agustín Tapia-Alba \\ Doctor en Estudios Sociales, Universidad Popular Autónoma del Estado de Puebla, \\ Puebla-México, juanagustin.tapia@upaep.mx \\ Cesairé Chiatchoua \\ Doctor en Desarrollo Económico, Instituto Politécnico Nacional, \\ Ciudad de México-México, cchiatchoua@ipn.mx
}

Cómo citar / How to cite

Tapia-Alba, J. A.; Chiatchoua, C. (2021). Análisis de la relación entre actividad empresarial y desigualdad económica. Revista CEA, v. 7, n. 14, e1597. https://doi.org/10.22430/24223182.1597

Recibido: 11 de diciembre de 2020

Aceptado: 16 de abril de 2021

\section{Resumen}

El objetivo de esta investigación fue analizar la relación entre el desarrollo de ciertas actividades empresariales y la generación de desigualdad económica, a la que se suman también la corrupción, la escalabilidad tecnológica, la innovación, las ganancias inmerecidas y el uso de recursos subvaluados. De acuerdo con el diseño de la investigación y las citas bibliográficas, se utilizaron fuentes de información secundarias; es decir, se aplicó una metodología analítica e interpretativa mediante el estudio de caso Poder de Mercado y Bienestar Social, de la Comisión Federal de Competencia Económica de México, el cual destaca la conformación de monopolios en el país y los efectos de sus prácticas comerciales. Los resultados muestran que aquellas empresas que se hayan visto más favorecidas serán las que presenten mejores resultados producto de factores diversos como la cultura organizacional, la forma en la que reaccionaron a las condiciones del entorno, su disponibilidad de recursos, las decisiones que toman, las medidas para reducir riesgos y el aprovechamiento de las oportunidades de negocio. El artículo señala, principalmente, el margen de sobreprecios que pagan los consumidores mexicanos cuando compran productos ofrecidos por las empresas indicadas en el estudio. Finalmente, la desigualdad es causada por los ciudadanos y sus organizaciones y ésta no es algo inherente o predeterminado de las sociedades a las que pertenecemos.

\footnotetext{
* Este artículo se deriva del proyecto titulado «Análisis de la relación entre actividad empresarial y desigualdad económica»
} y ha sido financiado con recursos propios. 
Palabras clave: economía social, política económica, perspectivas de crecimiento económico, globalización.

Clasificación JEL: D23, D24, D42 D63, O40, P1.

\title{
Highlights
}

- Los monopolios privados, principalmente, restringen la libertad económica del consumidor.

- Los sobreprecios que pagan los consumidores, producto de las decisiones de empresas representan un impuesto diferenciado.

- La desigualdad económica, como consecuencia de la actividad empresarial, aumenta por factores relacionados al entorno de las organizaciones.

- La desigualdad es una realidad en América Latina y es causada por los ciudadanos y sus organizaciones.

\begin{abstract}
This paper analyzes the relationship between the development of certain business activities and the generation of economic inequality, as well as corruption, technology scalability, innovation, undeserved revenue, and the use of undervalued resources. The authors used secondary information sources and an analytical-interpretive methodology to examine the study case described in the document entitled Poder de Mercado y Bienestar Social published by the Comisión Federal de Competencia Económica de México. Said document highlights the creation of monopolies in the country and the effects of their commercial practices. The results show that the companies that have received the greatest benefits from monopolies also present the best performance, which is produced by multiple factors (i.e., organizational culture, the way organizations react to the conditions of the environment, their resource availability, the decisions they make, their measures to reduce risks, and exploiting business opportunities). This article mainly refers to the surcharge margin paid by Mexican consumers when they buy products offered by the companies listed in the study mentioned above. Finally, inequality is caused by individuals and their organizations, but it is not inherent or predetermined in our societies.
\end{abstract}

Keywords: Social economics, economic policy, economic growth perspectives, globalization.

JEL classification: D23, D24, D42 D63, O40, P1.

\section{Highlights}

- Private monopolies mainly restrict consumers' economic freedom.

- Surcharges paid by consumers, caused by corporate decisions, represent a differentiated tax.

- Economic inequality, as a consequence of business activity, increases due to factors related to the environment of organizations.

- Inequality is a reality in Latin America, and it is caused by individuals and their organizations. 


\section{INTRODUCCIÓN}

El presente artículo destaca la posible relación que existe entre algunas actividades empresariales y las desigualdades económicas que ellas suscitan en el mundo (Bapuji, 2015). Para tal efecto, se debe identificar a partir del estudio de tres factores (corrupción, escalabilidad tecnológica e innovación; uso de recursos subvalorados; y ganancias inmerecidas) en qué casos las empresas pueden contribuir a un descenso o aumento de los niveles de desigualdad en economías de mercado (Conerly, 2018).

Asimismo, se revisan otros aportes al estudio sobre las implicaciones que el aumento de la desigualdad tiene sobre el funcionamiento de una economía de mercado (Goldberg, 2012). También se señala que la libre competencia es el insumo básico para el surgimiento y consolidación de instituciones inclusivas (Lin, 2007; Vail, 2009). De igual forma se reconoce la importancia de los estudios a partir de los años sesenta, relacionados con el comportamiento de las empresas y los resultados que estas ofrecen de acuerdo con la reacción que tienen frente a las condiciones de su entorno (Instituto Internacional de Gobernabilidad Barcelona, 1998).

El artículo destaca, además, la influencia que tiene la corrupción en el aumento de la desigualdad derivado de la distorsión que esa práctica ocasiona en el funcionamiento de una economía de mercado (Houle, 2018). Igualmente señala cuál es el impacto económico que la corrupción carga, primero, a la actividad empresarial, y posteriormente, a los consumidores, que son quienes terminan por pagar los costos asociados o derivados de su práctica. Por otro lado, se identifica la escalabilidad tecnológica como otro factor que permite destacar cómo las empresas desarrollan su ventaja competitiva y cómo ésta puede ser un precursor de la desigualdad (Neme-Castillo et al., 2021). En este punto existe un debate sobre la legitimidad en la formación de esa ventaja que se centra en la fuente de la escalabilidad tecnológica se distingue entre tipos de empresas y la posibilidad de escalar su tecnología.

Finalmente, se aborda la implicación que tienen los monopolios como precursores de desigualdad en economías de mercado y se señalan las dificultades que tienen los sistemas legales en combatir su formación y los costos que representa sobre la sociedad. Es conveniente señalar que este artículo no contempla los componentes morales y éticos asociados con este fenómeno. De lo anterior, el objetivo de este artículo es analizar la relación entre la forma en la que se llevan a cabo ciertas actividades empresariales y la desigualdad económica, la forma en la que la corrupción, la escalabilidad tecnológica e innovación pueden ser afectadas debido a la desigualdad ocasionada y, por último, cómo las ganancias inmerecidas y el uso de recursos subvaluados son los resultados más visibles de la mencionada desigualdad.

\section{MARCO TEÓRICO}

En este apartado se analizarán las variables de estudio para un mejor entendimiento, estos serán la desigualdad económica en un principio, después la corrupción y la escalabilidad. 


\section{La Desigualdad Económica}

La desigualdad económica se ha convertido, según Stiglitz (2015), en el principal problema económico en los últimos años. Las causas de esa problemática son varias, y algunas llegan al extremo de responsabilizar al sistema de economía de mercado de fomentarla, e inclusive de acrecentar sus niveles, en tanto aumenta la actividad económica en las sociedades modernas. El propósito principal de este artículo busca demostrar que el capitalismo, o, mejor dicho, la forma en la que éste se aplica puede generar condiciones que provoquen un aumento de los niveles de desigualdad en el mundo moderno.

La desigualdad económica es la creciente distancia que hay entre los grupos sociales con más ingresos en relación con aquellos de menores ingresos en una sociedad (Bennett \& Zitikis, 2015), o aquella propuesta por la Organización para la Cooperación y el Desarrollo Económico (OCDE, 2018), que sostiene que ésta es «el distinto reparto de los ingresos, los activos o el bienestar entre el conjunto de habitantes».

De lo anterior, se mencionan cinco causas que originan la desigualdad actual: la globalización, la irrupción de la tecnología, la distribución de la riqueza, los empleos y los salarios, los sistemas fiscales. Sin embargo, al hacer un análisis sobre cada uno de los elementos citados, se percibe que los mismos también pueden ser el origen de situaciones favorables para la realidad socioeconómica actual.

Es decir, parece ser que la desigualdad es resultado de cómo se aplica o evoluciona, en un determinado contexto social o económico, cada uno de los cinco factores enlistados anteriormente que generan una mayor desigualdad económica y no la condición per se de los mismos (Dada Hirezi, 2015). Retomado los trabajos de Piketty sobre la creciente desigualdad en el mundo los últimos 200 años, se observa en la conclusión que existe una brecha aparentemente pequeña entre el retorno del capital y la tasa de crecimiento de la economía, la cual tiene efectos poderosos y desestabilizantes sobre la estructura y la dinámica de la desigualdad social. Esta situación está lejos de terminar, menos aún con crisis como la del COVID-19. Becerril (2020) menciona que la pandemia ha generado altos niveles de desigualdad. Uno de los principales contrastes que se han vistos son los videos de las celebridades desde sus lujosos confinamientos, mientras los repartidores e integrantes de la gig economy tienen que salir a ganarse el sueldo del día.

\section{La corrupción}

Internacionalmente existe está Índice de Percepción de la Corrupción, publicado por Transparencia Internacional, que mide la opinión de la población de un país sobre las acciones que llevan a cabo sus autoridades. La importancia de ese instrumento es que da una idea aproximada sobre cómo la gente evalúa a sus gobiernos. Esa evaluación otorga una imagen que se relaciona y que tiene influencia en diversas actividades económicas y sociales en un determinado país (Baumann, 2019). Por lo anterior es común pensar que en aquellos lugares donde se perciben menores niveles de corrupción las autoridades tienen una mejor calificación entre sus habitantes; es decir, el nivel de confianza de los habitantes crece respecto a sus gobernantes. Cuartas Ricaurte (2016) concluye que un incremento de un punto porcentual en el índice de Gini (escala de medición de la desigualdad) reduce cerca de medio punto porcentual el porcentaje de personas que confían en el país. 
Es común pensar que la corrupción, o sus episodios más notables, sólo impactan la esfera gubernamental y su relación con la sociedad a la que gobiernan. El mencionado índice ofrece un dato revelador: a menor percepción de corrupción en un país, mayor es el grado de desarrollo de este, como lo menciona Reyes Hernández (2018) en un estudio sobre corrupción y desarrollo, donde encontró que, en 8 de 10 modelos de regresión, la corrupción reduce el desarrollo de los países. Por tanto, la corrupción afecta también la actividad empresarial y condiciona de forma importante sus resultados.

En la más reciente publicación del índice de Gini se aprecia que los primeros diez lugares son ocupados por países altamente desarrollados, siendo los tres primeros Dinamarca, Nueva Zelanda y Finlandia; mientras que los últimos diez puestos son ocupados por países pobres o que han sufrido un conflicto importante, ubicándose en los tres últimos lugares Sudán, Siria y Somalia (Banco Mundial, 2021). Lo anterior indica, de forma casi subconsciente, que es más fácil hacer negocios en Dinamarca y mucho más difícil hacerlos en Somalia. Una consecuencia de lo anterior lo representa la diferencia en la actividad económica que se lleva a cabo en un país o mercado, en comparación con otro. La corrupción tiende a disminuir el volumen de negocios o actividad económica que se desarrolla en los mercados.

En este punto, se pueden identificar cuatro áreas en las que la corrupción impacta de forma importante la actividad empresarial. La corrupción es un fenómeno que tiende a desplazar recursos económicos de un lugar deseable, en términos de la estrategia competitiva de las empresas, hacia otros más seguros y alejados de donde se practica la misma. Por tanto, la corrupción altera y condiciona la estrategia competitiva de la compañía, de forma tal que, una misma empresa deberá de adaptar sus planes de entrada en un mercado dependiendo de las condiciones que priven en el entorno de este.

En tanto, esas cuatro áreas en las que la corrupción condiciona las actividades de las empresas son: pago de impuestos y contribuciones, obtención de contratos, cumplimiento de leyes y reglamentos, y, por último, inversión. Cabe señalar que, para efectos del presente artículo, se aborda de forma sucinta cada uno de los cuatro elementos señalados pudiendo dedicar un artículo para cada uno de ellos. La corrupción tiene costos importantes sobre la actividad empresarial y es notable señalar que combatir la corrupción cuesta en términos del desarrollo de las empresas.

La corrupción representa un impuesto oculto que pagan los integrantes de una sociedad en el desempeño de sus actividades (Donadelli et al., 2014). Sin embargo, si se centra en el caso de las empresas, se ve que la corrupción tiene altos y ocultos costos sobre la operación de estas. Esos costos evitan que las empresas maximicen sus beneficios y, por tanto, los fabricantes de bienes o servicios deben considerar si absorben dichos costos o los transfieren a los precios de los artículos que ofrecen a sus consumidores. Hay que recordar en todo momento el concepto de impuesto oculto y las implicaciones que ello representa en sobreprecios por los bienes y servicios que ofrece el Estado o en aquellos que son ofrecidos al consumidor o usuario por medio de concesiones otorgadas por éste a empresas privadas (Costas \& Germà, 2000).

Conerly (2018) señala que las empresas pueden ser tanto responsables por el aumento de la desigualdad económica en un país como causantes de un descenso de esta. El mencionado autor señala tres puntos que influyen en la actividad empresarial y por tanto en los niveles de desigualdad 
que se pueden presentar en una economía: corrupción, escalabilidad de la innovación y uso de recursos subvaluados y mercados inmerecidos. Complementaríamos la afirmación anterior señalando que una misma empresa puede provocar ambas situaciones.

Conerly (2018) no lo menciona, pero puede identificar o relacionar sus argumentos con las propuestas de Acemoglu y Robinson en el sentido de distinguir entre organizaciones o empresas, para efectos de este artículo, inclusivas o extractivas (Acemoglu, 2012), de forma tal que las empresas que se desarrollan en ambientes mayormente inclusivos tienden a tener resultados que permiten identificarse con una reducción de las condiciones de desigualdad en las sociedades en las que se desenvuelven. Lo opuesto ocurrirá en aquellas mayormente extractivas.

\section{Escalabilidad de la Innovación}

Uno de los términos más difíciles de definir en el ámbito de los negocios es el de la escalabilidad. Sin embargo, es fácil identificar su efecto sobre la evolución de las empresas y su entorno. La escalabilidad es lo que ha permitido diferenciar a Amazon de cualquier tienda minorista o Uber del servicio público y local de taxis y distinguir entre Apple de Asus - la principal fabricante de computadoras personales en el área Asia Pacífico- (Medá, 2016). Es decir, el término se refiere a la característica que separa o hace crecer a una empresa con relación a sus competidores.

La escalabilidad, entonces, puede asociarse con la ventaja competitiva de las empresas que les permite obtener los mayores beneficios en el mercado, por lo que el tamaño cuenta. Las grandes corporaciones tienen mayores capacidades para imprimir escalabilidad a sus operaciones para desarrollar productos y servicios que les otorguen mayores ventajas con relación a sus competidores. Medá (2016) señala que la historia del término se puede remontar a los esfuerzos que IBM llevó a cabo para uniformar los requisitos tecnológicos que sus clientes debían cumplir para la instalación de redes de cómputo que la empresa les ofrecía hace más de treinta años.

En este orden de ideas entonces puede relacionarse también con la efectividad con la que las organizaciones o empresas pueden uniformar sus procesos y productos incluyendo elementos innovadores que permitan ofrecer mejores resultados con relación a la oferta que pueden hacer a sus consumidores. A pesar de que un término va ligado al otro - escalabilidad e innovación -, al parecer los mismos implican cosas distintas. La innovación quiere, más que otras cosas, velocidad en el acceso a nuevos productos y el tiempo en el que estas se hacen. La escalabilidad, en cambio, se enfoca en la confiabilidad (Ensighten, 2016).

Las aplicaciones del término escalabilidad en los negocios son diversas. Entre ellas se pueden mencionar las siguientes: la expansión de la empresa a mercados internacionales, el desarrollo de nuevas líneas de productos o áreas de negocio. Otros señalarán la reducción de los costos operacionales de la empresa al desarrollar economías de escala. Asimismo, existe también una diferencia en cuanto al entorno o ámbito en el que se desarrolla dicha escalabilidad: la escalabilidad interna y externa (Hoffman, 2019). Aquí se encuentra otro elemento que le da una mayor ventaja a las empresas grandes en comparación con las pequeñas.

La diferencia en cuestión de competitividad entre empresas, aumenta a medida que las empresas más grandes tienen mayores capacidades y gestionan el conocimiento en pro de desarrollar modelos 
de negocios que las hagan obtener ventajas competitivas (Toro-García et al., 2020). La escalabilidad y la innovación estarán, entonces, al servicio de aquellas organizaciones que se encuentren en una mejor posición competitiva en un determinado ambiente de negocios. Es así como las ventajas competitivas de Uber son causa y producto que le permite a la empresa vencer, en forma creciente, en el mercado del transporte público de pasajeros en las ciudades en las que compite.

Como puede suponerse, las condiciones a las que se ve expuesta una empresa en el entorno de negocios en el que participa son los factores que condicionarán la escalabilidad externa de la empresa. Compañías como PetroChina, General Electric y Exxon eran las empresas más valiosas en el mundo en términos de capitalización hace diez años. Esas empresas han sido reemplazadas por los gigantes de las plataformas digitales como Apple, Amazon, Alphabet- Google- y Microsoft (Hoffman, 2019). Lo anterior ha ocurrido independientemente de la eficiencia operativa y en los modelos de negocio bajo las cuales funcionan las empresas desplazadas.

Para Moser (2018), existen diversos factores que influyen en el desarrollo de los modelos de negocio que usarán las empresas. De la misma forma, esos factores también determinan la escalabilidad del sector industrial y comercial donde se desempeña la firma. Factores como la cultura, la legislación aplicable, los competidores y la tecnología también hacen su parte para provocar cambios y adaptaciones con tal de aumentar la competitividad y rentabilidad de las compañías en un entorno cada vez más hostil.

Los inversionistas en empresas que desarrollan sus actividades en áreas tecnológicas han descubierto que el secreto que tienen en común las plataformas digitales señaladas es la escalabilidad externa de sus operaciones de negocios. Esa escalabilidad es, o debería ser, resultado de la innovación de quienes colaboran con la empresa. La escalabilidad, como se anotó anteriormente, es el factor diferenciador en sus operaciones e indica que aquellos sectores industriales donde hay involucrada mayor tecnología, también generan más valor agregado y requieren de menores volúmenes de capital financiero, pero mayores capacidades intelectuales de sus trabajadores.

Stiglitz (2015), sin embargo, no es tan optimista en cuanto al poder creciente que han adquirido los gigantes tecnológicos, también conocidos como GAFAT - Google, Apple, Facebook, Amazon y Twitter-, ya que señala que se han convertido en la principal amenaza a la libre competencia en la economía estadounidense (Graham, 2018). Más aún, señala que esas empresas han aprovechado indebidamente - ya que no pagan derechos de uso - la inversión pública hecha por el gobierno estadounidense en el desarrollo del DARPA/ ARPANET, el antecedente de la actual Internet, cosa que no ocurre con muchas otras empresas que sí tienen que pagar por usar la infraestructura que requieren para desarrollar sus actividades.

En cuanto a la innovación, se define, según (Alpuche, 2017), como un proceso que implica una modificación de una determinada situación en aras de lograr un mejoramiento en procesos, productos o servicios ya sea en una organización o en una comunidad. Para el caso de las empresas prevalece un proceso de destrucción creativa donde el empresario es el agente clave de la innovación, y donde las rutinas y la evolución organizacionales juegan un papel crucial para el cambio económico, y, por lo demás, persiste la existencia de una brecha tecnológica entre los países económicamente avanzados y los atrasados. 
De lo anterior se puede decir que la misma es el factor diferenciador por excelencia entre las empresas en la actualidad. La capacidad de innovar de las organizaciones depende de factores externos, pero primordialmente internos. Las organizaciones que hacen de ésta un componente importante en la gestión de sus operaciones cuentan con mejores oportunidades de maximizar su ventaja competitiva. Sin embargo, no es recomendable pensar que la innovación pueda considerarse una moda, ya que quien lo piense así corre el riesgo de tener una concepción obsoleta de la forma en la que las organizaciones deben competir en el entorno de negocios.

De la misma forma, el neoinstitucionalismo también distingue entre las vías productiva e improductiva. Ésta última, según North (1993), «...las economías cuyas instituciones incentivan el estancamiento pueden persistir, en función de las características del ambiente humano y de las formas en que los humanos lo interpretan». La influencia del entorno en el ámbito empresarial es crucial, ya que el mismo condiciona de forma importante el actuar de la empresa, que inicialmente debe adaptarse y crecer.

\section{METODOLOGÍA}

Para el alcanzar el objetivo de este documento, se usaron fuentes secundarias, pues se pretende profundizar y ampliar el conocimiento sobre la situación prevalente en la actividad empresarial mexicana y su relación con la desigualdad económica. Se realizó una revisión bibliográfica exhaustiva y actualizada sobre los informes de las estadísticas oficiales en torno a componentes como la corrupción, la escalabilidad de la innovación y el uso de recursos subvaluados en México. Una vez realizada la revisión bibliográfica se procedió a interrelacionar la información obtenida para analizarla, así como deducir la presentación adecuada de las variables objeto de estudio.

La búsqueda de bibliografía se centró en encontrar evidencia que se presenta en el artículo sobre tres elementos. Uno, el efecto que causan las prácticas de corrupción sobre la actividad empresarial y sus consecuencias en materia de aumento de costos que sufren las empresas al desarrollar sus actividades, sobre todo aquellas que relacionadas con la obtención de contratos públicos y que, como se indica en el documento, pueden generar impuestos ocultos al contribuyente.

Posteriormente, en el caso de la escalabilidad tecnológica se incluyen evidencias de cómo la inversión que hace México en materia de ciencia y tecnología contribuye a generar mayores condiciones de desigualdad debido a que el país va en contrasentido con relación a los países más avanzados del mundo en materia tecnológica. Asimismo, se indican cuáles son las tendencias en materia de escalabilidad tecnológica y se incluye la definición del término, que explican la creciente competitividad de las empresas más importantes del mundo.

Por último, se incluye un caso de competencia monopólica para ilustrar la obtención de ganancias inmerecidas a partir del uso de recursos subvaluados en la obtención de ganancias inmerecidas típicamente relacionadas con ese tipo de competencia económica. En concreto, se ilustra cómo la manipulación de la tasa de interés que el Banco de México emitía para pagar el rendimiento de bonos de deuda del gobierno ofrecía ganancias adicionales a los bancos comerciales que monopolizaban la compra de los bonos mencionados. 


\section{RESULTADOS}

\section{La actividad empresarial y su relación con la desigualdad económica}

Aquí se aborda el tema empresarial y su relación con la globalización. Aquellas empresas que se hayan visto más favorecidas por el proceso de globalización serán las que presenten mejores resultados producto de una diversidad de factores tales como la cultura organizacional, la forma en la que reaccionaron a las condiciones del entorno, su disponibilidad de recursos, las decisiones que toman, la reducción del riesgo y el aprovechamiento de las oportunidades de negocio que tienen.

Es aquí donde cabe hacer un señalamiento puntual: el capitalismo no es el causante de la creciente desigualdad económica que enfrenta el mundo moderno. Por el contrario, es el responsable de la mayor creación de riqueza económica que el mundo haya visto en toda su historia. El producto interno bruto (PIB) mundial, de 2010, era, de 11.8 billones de dólares, mientras que en 2018 era de 80.2 billones de dólares (Worldometers, 2019). La riqueza, al igual que la pobreza, no se distribuye de la misma forma en todos los países del mundo. Algunos crecen más que otros debido a la forma como se implanta el capitalismo en una sociedad en comparación con otras. Algunos crecen mucho más que otros, y esa realidad se debe más a cómo se implanta el capitalismo en una sociedad en comparación con otras.

En este punto se identifica una característica fundamental de toda empresa exitosa: la capacidad que tenga para diseñar su estrategia competitiva de acuerdo con el entorno que le corresponde enfrentar. Lo anterior es algo que toda empresa global sabe: diseñar una estrategia distinta y específica de acuerdo con sus capacidades y objetivos sin olvidar que los mismos deben adaptarse a un tiempo y lugar específicos. Es menester recuperar lo dicho por (Conerly, 2018) en el sentido de las tres fuerzas o componentes: corrupción, escalabilidad de la innovación y uso de recursos subvaluados y mercados inmerecido, que puedan aumentar o disminuir el nivel de desigualdad en una sociedad determinada.

\section{La corrupción y su relación con la desigualdad}

Según Albarrán (2018), el costo de la corrupción, en términos económicos, pudo alcanzar, por su carácter oculto, entre un $5 \%$ y $10 \%$ del PIB en México en 2018. Esos porcentajes representan hasta 2.3 billones de pesos al año. Además, resalta que esa cifra es cinco veces mayor al promedio de los países que integran al organismo. La misma organización señala que la corrupción se traduce en distintos efectos que perjudican la evolución económica de un país; a saber: merma de la competitividad de las empresas y del país; políticas públicas deficientes; afecta la confianza en los mercados; y uso ineficiente de los recursos. Asimismo, México, según la OCDE, es su integrante menos transparente.

La OCDE destacaba, en 2017, que los recursos relacionados con la práctica de la corrupción en una economía se pierden, ya que los mismos no se invierten en áreas que privilegien el interés público, elemento que debiera guiar la actuación de todo gobierno en una sociedad moderna y democrática. El destino de esos recursos es desconocido, ya que los mismos terminan en las manos o cuentas bancarias de alguien que no puede hacer pública su posesión. Es decir, la corrupción redirecciona 
una importante cantidad de recursos financieros que terminan en la clandestinidad. Casi nadie sabe dónde terminaron esos 2.3 billones de pesos al año.

La dinámica señalada que causa la transferencia de 2.3 billones de pesos al año, en el caso mexicano, tiene diversos orígenes y destinos que no necesariamente son los mismos. Es decir, si una empresa ofrece un soborno, que es un acto ilegal, a los funcionarios fiscales con tal de eliminar o minimizar el pago de algún impuesto, el dinero pagado por el soborno va a la cuenta del funcionario que lo recibe. Dicho funcionario no puede informar a sus superiores que ha recibido ese pago, por tanto, deberá ocultarlo.

El funcionario tiene dos opciones: esconderlo dentro o fuera del país. Si lo hace dentro, quizá lo invierta en alguna actividad productiva o instrumento financiero que podría reportarle algún beneficio debido a las utilidades que esa actividad o instrumento genere. En caso contrario, si el funcionario lo invierte fuera del país, entonces los beneficios generados por esa inversión no impactarán de forma alguna en la economía nacional. Sin embargo, la evolución de los mecanismos para la rendición de cuentas en las democracias modernas tiende a hacer cada vez más complicado conservar los recursos producto de un soborno dentro de la economía local, por lo que la opción de llevarlo fuera del país va ganado terreno.

Indiferente de la decisión que tome el funcionario que recibe un soborno, sobre invertirlo dentro o fuera del país, hay algo que no cambiará: una empresa, al no pagar los impuestos que debe, impide que ese dinero que se debe recaudar llegue a las arcas públicas, impidiendo que se invierta en educación, salud o construcción de infraestructura que mejore las condiciones competitivas de un país. La empresa que paga el soborno sabe que puede minimizar sus costos incumpliendo las leyes locales y estará tentada en decidir que sus consumidores paguen, en el precio de sus productos, lo invertido para reducir el costo de sus obligaciones.

El consumidor, en el ejemplo anterior, es quien pierde más debido a que como contribuyente recibirá menores servicios públicos, debido a que el gobierno recauda menos impuestos de los debidos, e inclusive podría pagar costos ocultos en los productos que compra, ya que la empresa decidió trasladar el mismo a sus clientes. Hay que señalar que, debido al ciclo económico básico, todos son productores y consumidores en la vida diaria.

Además, la empresa tendrá la percepción de que podría cometer otros abusos contra sus consumidores debido a que sabe que el gobierno incumplirá su responsabilidad de proteger los intereses de las personas a las que gobierna. De forma tal que las compañías deberán decidir si se adaptan al esquema de pagar sobornos a cabo de beneficios en el pago de sus obligaciones o cumplir con sus responsabilidades con los costos y beneficios que ello represente. La corrupción institucionalizada le ofrecerá un estímulo importante a quienes estén interesados en llevar a cabo actividades empresariales, en el sentido de que puede ser buena idea no hacer lo que es debido.

Los 2.3 billones de pesos no se perdieron, sólo se transfirieron de los bolsillos de empresas y consumidores hacía aquellos de algunos funcionarios que decidieron incumplir con su obligación como servidores públicos. La corrupción es un estímulo para la irresponsabilidad de los funcionarios públicos y da una lección sobre cómo evadir el cumplimiento de las obligaciones fiscales a las empresas, al tiempo que perjudica, en dos sentidos, los intereses de los consumidores. La corrupción 
contribuye a generar empresas y organizaciones extractivas y es uno de los peores enemigos de las organizaciones inclusivas.

En este punto resalta una cuestión fundamental: la sociedad en su conjunto debería combatir la corrupción, independientemente de sus implicaciones legales y morales, bajo el argumento de que la misma cuesta dinero y es menester aprovechar de mejor forma de los recursos de los que dispone. Una mejor asignación de recursos hará más eficiente el desarrollo de las actividades económicas en un determinado lugar. México podría usar mejor esos 2.3 billones de pesos al año ya que esos recursos podrán destinarse a actividades que generen beneficios que puedan ser visibles en cualquier esfera de la actividad económica.

Esos recursos podrán destinarse a actividades de consumo, las mismas que generarán un mayor pago de dinero al fisco federal o estatal. Las empresas reducirán sus costos de operación debido a que ya no habrá necesidad de pagar sobornos a las autoridades corruptas. Los precios de los bienes y servicios ya no cargarán con la parte oculta que representó el otorgamiento de pagos indebidos a los funcionarios públicos. La economía nacional podría crecer en dimensiones similares a la proporción del PIB que se desvía por prácticas de corrupción. El impacto de esa práctica va de 10\%, según la OCDE, (OCDE, 2017) hasta el 18\% de ese indicador, según la Asociación Mexicana de Profesionales de Ética y Cumplimiento (AMPEC) (Lara, 2018), una asociación poco conocida por sus estudios o aportaciones en materia de combate a la corrupción en el país.

Existe un componente importante que se ve afectado por la corrupción: la inversión. ¿Cómo se ve afectada ésta por aquella? En una primera instancia hay que señalar que el carácter oculto de las prácticas de corrupción evita que pueda haber una relación evidente entre los elementos señalados. Es decir, al ser muy complicado rastrear cuándo se comete un acto de corrupción, cuánto dinero hay involucrado en esa transacción y dónde se depositan esos recursos o el uso que se les dio, es, asimismo, difícil ver qué implicaciones puede tener en los flujos de inversión que pueden llegar o salir en una economía nacional.

Algunas publicaciones señalan el efecto de la corrupción sobre la inversión, entre ellas destacan cómo la globalización también trajo consigo que la corrupción se extendiera a sitios que hasta hace poco se creían ajenos a la misma, tales como Estados Unidos o Europa (Elliott, 2001), mientras Johnson (2001) destaca la relación entre la corrupción y la función política y sus efectos en materia de decisiones económicas y de inversión. Por último, el efecto de esa práctica en materia de inversión y crecimiento económico tiene una relación inversamente proporcional (Mauro, 2001).

Sin embargo, en ciertos casos se aprecia que la corrupción puede desincentivar la inversión privada debido a que los inversionistas aprecian que el retorno de esta puede ser menor (Everhart et al., 2009). En contraste, las inversiones en el sector público, en el caso de las empresas estatales, pueden estar orientadas por consideraciones adicionales o sustitutivas a la obtención de utilidades. De hecho, en múltiples casos, ese tipo de inversiones, y quienes las encabezan, pueden ser designadas de acuerdo con su cercanía con los centros de poder en donde se toman las decisiones políticas más importantes.

En este último caso, hay evidencias que prueban que la inversión pública y la corrupción tienen una correlación positiva. Dicha relación descubre que las inversiones que se hacen en el sector público 
pueden generar mayores oportunidades para las prácticas rentistas y la malversación de fondos públicos (Keefer \& Knack, 2007). Lo anterior representa una desviación del interés público, misma que encubre una práctica de corrupción patrocinada por un sistema político que se ha encargado de dilapidar los fondos públicos a cambio del enriquecimiento de los grupos de interés más cercanos al poder en distintos países.

\section{Escalabilidad de la innovación}

Una de las preocupaciones más importantes que deben tomar en cuenta los líderes de las organizaciones empresariales es la forma en la que adoptarán la innovación en la gestión de sus negocios. Para tal efecto, existen diversas alternativas que se puede identificar como el proceso mediante el cual una organización ordena y dirige sus recursos (humanos, materiales y económicos) con el objetivo de aumentar la creación de nuevos conocimientos, generar ideas que permitan desarrollar nuevos productos, procesos y servicios o mejorar los ya existentes, y transferir ese conocimiento a todas las áreas de actividad de la organización (Falcone-Treviño et al., 2018). Lo anterior es relevante considerando los costos y beneficios que están involucrados en el desarrollo de proyectos de innovación y cómo los mismos impactan sobre el funcionamiento de las empresas.

Es decir, si consideran los criterios que sirven para distinguir los tipos de innovación en el mercado, se puede señalar que la misma se divide en innovación radical, cuyo objetivo tiene el desarrollo de productos que podrían ser considerados como altamente innovadores, mismos que requieren de mayores presupuestos de inversión en materia de recursos financieros, tecnológicos y humanos. Por lo tanto, este tipo de innovaciones implican mayores riesgos, donde sólo un número reducido de empresas puede comprometer a solventar las demandas de inversión de los recursos requeridos para su desarrollo.

El otro tipo de innovación es la incremental. En este caso, los montos requeridos para llevar a cabo su desarrollo e implantación en el mercado son menores en comparación con la radical. De la misma forma, su nivel de riesgo también es menor $y$, debido a eso, hay más empresas que están en posibilidades de participar en este tipo de actividades. Por consecuencia, la innovación, los riesgos y las posibilidades que ofrece son distintos dependiendo del tipo de organización, mercado y condiciones del entorno, las que indican que las compañías deberán encontrar su lugar en el mercado y tomar ventaja de su posición correspondiente.

De forma adicional se puede mencionar que habrá que considerar cuál es el objeto sobre el que se desarrolla la innovación. En este renglón, también existen dos formas de innovar. Los productos que son modificados en la primera de esas dos formas de innovar son objetos que se ponen a disposición de los consumidores en el mercado. Este tipo de innovación se conoce como Innovación de Productos. La otra clase tiene, en cambio, la intención de modificar el know-how, o Innovación en procesos de las empresas, para que, producto del tipo de cambio que se aplique en la organización, las empresas vean sus costos de producción estabilizarse o descender para ofrecer productos o servicios más eficientes.

Es relevante considerar que el tipo o enfoque que den las empresas a sus inversiones en materia de innovación - radical o incremental; de producto o proceso - deberán considerar que la radical y de proceso implican niveles de riesgo e inversión mayores en comparación con la incremental o de 
producto. Asimismo, si el nivel de riesgo es mayor, también pueden ser los beneficios económicos que puede generar. Es decir, si una empresa desarrolla innovaciones de tipo incremental o de proceso, la misma se convierte en la primera en desarrollar o fabricar un producto. Es decir, puede obtener una first-mover advantage (ventaja del primer movimiento, en este caso ventaja en ser el primero en fabricar el producto). Los beneficios financieros pueden ser mayores si la innovación es radical o de proceso.

Son tres los principales beneficios que puede obtener una empresa cuando se convierte en la primera en desarrollar un producto o crear un nuevo proceso de fabricación: liderazgo tecnológico, control de los recursos en una determinada industria y lealtad a la marca que se desarrolla en los consumidores. Por otro lado, las desventajas de la misma destacan los menores costos que deberán invertir los competidores para ofrecer su versión del producto en el mercado, los competidores habrán aprendido la lección de la first mover y podrán revertir o mejorar sus estrategias de competencia con base en la mejora en decisiones relacionadas con la inversión, la adaptación tecnológica y la experiencia en el mercado (Corporate Finance Institute [CFI], s. f.).

Lo anterior conduce, entonces, a una pregunta central con relación a los objetivos del presente documento: ¿Cómo se relaciona la innovación empresarial y la desigualdad económica? Una respuesta potencial a esa pregunta indicaría, con base en lo discutido anteriormente, que las empresas más innovadoras tienden a ampliar su ventaja competitiva con relación a las que no reconocen a la innovación como un elemento fundamental, ya sea por falta de recursos financieros o tecnológicos o de una visión a largo plazo, en la gestión de sus organizaciones. Es decir, aquellas empresas que no innoven estarán en desventaja con relación a aquellas que sí lo hagan.

Las repercusiones de la existencia - y crecimiento - de las ventajas competitivas entre empresas pueden generar condiciones que están relacionadas con el surgimiento tanto de menores como mayores niveles de desigualdad en la macroeconomía. Es decir, el aumento de competitividad de las compañías Silicon Valley, puede aumentar los niveles de desigualdad en San Francisco, lugar en el que estas se desarrollan. Sin embargo, también las empresas pueden impulsar innovaciones que contribuyan a mejorar las condiciones de vida de la gente en diversos lugares, así como de investigaciones en el ámbito médico y tecnológico (Glennie \& Gabriel, 2017).

En el ámbito macroeconómico se distingue la realidad de los discursos en materia de inversión dirigida a la innovación empresarial. En el caso mexicano, se creó la Ley de Ciencia y Tecnología y sus reformas de 2009, 2012 y 2015; con respecto a las actividades que lleva a cabo el Consejo Nacional de Ciencia y Tecnología (Conacyt) para promover la investigación científica y tecnológica, la cual busca generar mejores condiciones para la innovación en el país y el otorgamiento de estímulos fiscales a las empresas que inviertan en proyectos tecnológicos e innovadores, la realidad del país indica lo siguiente:

México invierte el $0.5 \%$ de su PIB en investigación y desarrollo. En términos de PIB por Paridad de Compra (PPP), el país destina 11.5 mil millones de dólares. El 37\% de esa suma la invierte el gobierno, el $30 \%$ las empresas, el $26 \%$ las universidades y el resto organizaciones privadas no lucrativas. Cuenta con 244 investigadores por cada millón de habitantes. El 67\% de sus investigadores son hombres (Organización de las Naciones Unidas para la Educación, la Ciencia y la Cultura [UNESCO], s.f.). Lo 
anterior sirve para demostrar que el papel que deben jugar los empresarios en México en materia de inversión y en materia tecnológica debe ser mucho más importante.

Una característica fundamental que permite diferenciar un país desarrollado de otro que no lo es, es la proporción del PIB que destina a ciencia y tecnología. Es decir, entre mayor sea la contribución que un país al desarrollo de la innovación, mejores serán sus condiciones de vida. Aquí se puede distinguir una cifra mágica que muestra la frontera entre un grupo de países otro: cuando se destina más del $1 \%$ del PIB en ciencia y tecnología, o se supera esa proporción en países con economías en transición como Brasil, Estonia y Eslovenia, estos se asemejarán a países desarrollados. En ningún caso se encuentran países desarrollados que inviertan menos del $1 \%$ de su PIB en R\&D (UNESCO Research \& Development).

El informe de la UNESCO destaca otro punto fundamental: ningún país desarrollado en el mundo tiene la misma distribución de inversionistas en comparación con el caso mexicano. Es decir, en ninguno de esos casos se aprecia que el mayor inversionista en materia tecnológica sea el gobierno o las universidades, ese papel lo desarrollan las empresas privadas. Inclusive en algunos países que anteriormente fueron economías centralmente planificadas (Rusia, China, República Checa, Montenegro, Polonia y Kazajistán) se repite la misma tendencia. México hace lo mismo que India, Irán o Argentina en materia de inversión tecnológica (UNESCO, s.f.).

\section{Uso de recursos subvaluados y mercados inmerecidos}

La desigualdad económica, como producto de la actividad empresarial, puede disminuir cuando se promueve y existe una libre competencia entre los actores económicos (Ortiz Baquero \& Solano Osorio, 2016). Es decir, cuando las condiciones son más propicias para el libre intercambio de bienes y servicios en el mercado, las interacciones entre oferentes y demandantes serán más productivas, o benéficas, para los mismos. De forma tal que, entre mayores beneficios aprecien los agentes que obtienen en sus intercambios, menores serán las condiciones desiguales en las que se desarrolle la actividad económica en su conjunto.

La libre competencia permite que los consumidores tengan acceso a mejores productos, mismos que son resultado de inversión en materia de innovación. De la misma forma, la empresa hubo de invertir porque compite contra otras por la preferencia del comprador de sus artículos. También el gobierno cumple con su función de supervisión de la actividad económica en el sentido de que la economía crezca debido a las interacciones entre vendedores y compradores. Aquí, los precios se fijan libremente y los involucrados en las relaciones de intercambio no tienen el poder de controlar el mercado.

Un ejemplo de la dinámica que permite el aumento de la desigualdad económica en las sociedades modernas, producto de la explotación de un recurso subvaluado, es el caso de los salarios que perciben los trabajadores, los cuales tienen costos o precios distintos en el marco comparativo entre empresas ubicadas en distintos países. ¿Por qué el salario, para trabajos iguales, es distinto si es comparado con diversos países? La respuesta se puede encontrar en las condiciones de oferta y demanda que existen en el mercado laboral. En aquellos lugares donde el salario es mayor, se pueden apreciar ciertas condiciones que no se presentan de forma similar en otros mercados laborales. 
La existencia de mecanismos que protejan - o mejoren - las condiciones laborales y los derechos de los trabajadores a través de la participación de sindicatos y oficinas gubernamentales, así como laborar en una economía en expansión puede aumentar la demanda de trabajadores; por lo tanto, los salarios pueden aumentar debido a las mejores condiciones laborales existentes en un país a diferencia de otros. De la misma forma, una economía con altas tasas de crecimiento demanda una mayor cantidad de trabajadores en comparación con la oferta de estos, lo cual provoca que el precio de la mano de obra aumente. De la misma forma, las condiciones tecnológicas y la disponibilidad de mano de obra calificada son factores que permiten el crecimiento de los salarios en las economías modernas.

Por el contrario, si una economía no crece de forma importante, el Estado puede perder buena parte de su capacidad reguladora en materia laboral. De forma adicional, cuando la protección a los trabajadores se ve reducida en virtud de la existencia de reglamentos que favorecen la desaparición del estado del bienestar y favorecen la reducción de la carga laboral para las empresas, además de existir una condición de sobreoferta de trabajadores que demandan un empleo, tenderá a la erosión de los salarios y los beneficios laborales que serían aplicados en el caso del mejoramiento del entorno económico.

El principal enemigo de las economías de mercado no lo representa la economía planificada o socialista. Su principal opositor es la aparición de monopolios y oligopolios, los mismos que restringen la libertad económica y ofrecen ganancias inmerecidas a los propietarios de ese tipo de empresas. Esas ganancias pueden considerarse como el origen de la desigualdad económica. Las mismas son resultados de un mecanismo de apropiación de rentas que no son producto de la competitividad, innovación o visión empresarial del productor, sino de la apropiación de beneficios producto de una distorsión del mercado.

La actuación de los monopolios en una economía de mercado, cuando no son propiedad estatal, reduce la capacidad coercitiva del Estado con relación a los agentes económicos. Esa pérdida de poder estatal se refleja en diversas formas: disminución de la capacidad del Estado para supervisar la actividad económica; imposibilidad para hacer cumplir las leyes en contra de los monopolios; y menor recaudación fiscal debido a que ese tipo de empresas tienen formas de condicionar a los gobiernos para que las leyes fiscales se adapten a los intereses de las empresas, yendo en contra del interés común.

En el diseño de la política comercial de algunos países, los grupos de interés, en la búsqueda de maximizar sus beneficios, presionan a los gobiernos (Krugman le llama competencia electoral) para aplicar prácticas proteccionistas que son apoyadas por buena parte de la población, aunque las mismas van en contra del interés de los consumidores (Krugman, 2006). Un ejemplo de lo anterior es la imposición de aranceles o mayores restricciones a las importaciones con el objetivo de proteger a un reducido grupo de productores locales, aun cuando dichos aranceles o restricciones impedirán la importación de bienes que podrían tener menores costos y ser productos más eficientes con relación a los fabricados localmente.

Además, la formación de un monopolio, en muchos casos, enmascara prácticas de corrupción en el ámbito económico. Un estudio sobre corrupción publicado por la OCDE (2015), indica que existen algunos sectores industriales que son más proclives a las prácticas de corrupción, donde hasta dos 
terceras partes de este tipo se concentran en cuatro sectores industriales: minería (19\%), construcción (15\%), transporte (15\%) y comunicación e información (10\%). No es difícil imaginar, por tanto, que esas maniobras de corrupción han servido para que los empresarios de los sectores mencionados obtengan contratos o condiciones de negocios preferenciales a sus intereses.

En México, la actividad monopólica es intensa y controla sectores industriales a través de un conjunto de prácticas que afectan el interés de los consumidores. "América Móvil, Bimbo, Cementos Mexicanos, Coca-Cola Femsa, Grupo México, Gruma y Televisa dominan sus respectivos mercados a nivel nacional. Su preponderancia no las vuelve monopolios por sí mismas; aunque implica riesgos a la sana competencia económica por la posibilidad que tienen de imponer condiciones de mercado» (Flores, 2018). Hay que señalar que, en México, la Comisión Federal de Competencia Económica (Cofece), instancia encargada de eliminar la formación de monopolios, ha sido incapaz de eliminarlos debido a la complejidad de las disposiciones en la materia.

La señalada complejidad de las disposiciones antimonopolio se ilustra en la naturaleza, características y diferencias entre las prácticas monopólicas absolutas y relativas. En el primer caso, este tipo de prácticas se caracterizan por la capacidad que tienen algunas empresas de acordar condiciones relativas a la colusión de los participantes de un mercado para restringir, controlar y beneficiarse del uso exclusivo de un mercado. Las segundas, implican la capacidad de uno o varios competidores para acaparar el mercado (Flores, 2018).

Las actividades monopólicas de algunas empresas impactan en el aumento de los indicadores de desigualdad, debido a que las prácticas de ese tipo pueden subir los precios de los bienes y servicios que ofrecen por motivos que no tienen que ver con la interacción de los agentes económicos producto de relación de oferta y demanda asociados a condiciones de libre concurrencia en el mercado. Los monopolios modifican los precios de sus productos de forma discrecional, perjudicando el interés del consumidor y apropiándose de ganancias inmerecidas.

En un estudio publicado en 2018, se consigna que los efectos de las prácticas monopólicas de algunas empresas dominantes en México han causado que los hogares mexicanos paguen un sobreprecio de hasta $98.23 \%$ al momento de consumir bienes de consumo final (Comisión Federal de Competencia Económica [Cofece], 2018). Por supuesto, lo anterior impacta de forma diferenciada de acuerdo con los grados de desarrollo en el país y los niveles de ingreso de los distintos grupos que conforman la sociedad mexicana. Los sectores menos favorecidos serán los que paguen un costo mayor en relación con su ingreso, en relación con los demás. Esta es la muestra más clara del efecto de la desigualdad en una sociedad moderna.

Los monopolios privados, principalmente, restringen la libertad económica del consumidor. De hecho, según la misma Cofece, esa restricción se traduce en una menor elasticidad de precios de los productos que adquiere buena parte de la población en México. Por lo anterior, la desigualdad aumenta como producto de una menor libertad de elección que tienen los consumidores, especialmente aquellos que cuentan con menores ingresos. Esa menor libertad económica se traduce en una menor cantidad de oferentes de un producto, por ejemplo, pan blanco, de forma tal que la única empresa que vende ese producto en el país lo puede hacer bajo las condiciones de oferta, precio y características que la empresa decida. Como se mencionó, el impacto de la competencia monopólica tiene efectos diferenciados en México, aquí una muestra: 
Para el decil con menor ingreso, las pérdidas son de hasta $30.9 \%$ del ingreso mensual, equivalentes a 841 pesos. En el caso del decil más alto, las pérdidas ascienden a 2,137 pesos, que el equivalen al 5.7\% del ingreso mensual. (SinEmbargo, 2018)

De lo anterior se concluye que los sobreprecios que pagan los consumidores, producto de las decisiones de empresas que en la práctica funcionan como monopolios de imponer precios sin presión de otros consumidores, representa un impuesto diferenciado que afecta más a los consumidores de menores ingresos, por tanto, es necesaria la intervención de las autoridades antimonopolio para evitar que esas empresas sigan ampliando las márgenes de desigualdad en la economía. Los monopolios van en contra del interés público y el tipo de prácticas que desarrollan son rentistas.

Es recomendable que el marco legal se ajuste a la realidad comercial para que la detección y sanción de prácticas monopólicas sean más efectivas y, de esa forma, la protección a los intereses de los consumidores y la libertad económica responda efectivamente al interés público. Lo anterior no es sencillo, ya que, como se mencionó antes, los grupos de interés, en este caso las empresas monopólicas, cuentan con mecanismos de defensa más efectivos que el consumidor. Esos grupos pueden usar argumentos muy efectivos como la defensa de la economía de mercado, la competitividad e incluso criterios relacionados con el nacionalismo para evitar que las autoridades afecten sus intereses.

Es conveniente señalar que la defensa a los intereses del consumidor en México, aunque debiera mejorar, es superior a otros países. Un ejemplo de lo anterior se puede ilustrar en el caso de la existencia de organismos como la Procuraduría Federal del Consumidor (Profeco), instancia que funciona como mediador y autoridad en materia de mediación, protección y sanción a empresas que usen su poder de mercado en contra de quien adquiere un bien o paga un servicio en el país. Lo anterior es de resaltar, ya que este tipo de organismos no existen en los Estados Unidos, ni en otros países.

Existe otra esfera de actividad donde los monopolios, en el caso mexicano, obtienen ganancias inmerecidas, las mismas que van en contra del interés público; el pago de contribuciones y el cumplimiento de las sanciones a las que son acreedores por su actividad en contra de la libre competencia. Un caso relevante en la materia corresponde a una multa impuesta por la Cofece, previa investigación del Instituto Federal de Telecomunicaciones (IFT), en contra de Radiomóvil Dipsa y Telcel, por prácticas monopólicas y que equivalía, en 2018, a poco más de 12 mil millones de pesos. Sin embargo, la empresa negoció la conmutación de la sanción de forma tal que

El problema de ese acuerdo es que la autoridad procompetencia se conformó con un conjunto de medidas que en realidad no significaban un esfuerzo real de Telcel, pues en realidad eran medidas que ya habías sido descontadas por el mercado. (Flores Ramírez, 2018)

Como se aprecia, el comportamiento de las empresas monopólicas es completamente distinto al demandado por personas físicas y morales en sus relaciones de mercado. Es decir, si un contribuyente pequeño o mediano incumple con alguna disposición fiscal o en materia de libre concurrencia al mercado, se hace acreedor a una sanción. En condiciones normales, esa sanción no está sujeta a ser conmutada derivada del poder de negociación de la empresa, por lo tanto, la multa debe pagarse y 
deberá evitar repetir la acción que la ocasionó. Lo sorprendente en este caso es que la empresa cometió las faltas que le hicieron acreedora de la sanción, la cual no quiere pagar y, para evitarlo, promete portarse bien en el futuro. A los monopolios les incomoda, por decir lo menos, el cumplimiento de la ley.

A continuación, se analiza un caso que incluye los tres elementos que pueden generar mayor desigualdad producto de las prácticas que llevan a cabo ciertas empresas u organizaciones, y cuyas consecuencias pueden generar costos que son pagados por consumidores y/o contribuyentes. El caso en cuestión trata sobre la colusión, denunciada por la Cofece, que han llevado a cabo algunas autoridades financieras con bancos que operan en el mercado mexicano y en el extranjero para generar beneficios a partir de la especulación financiera.

El caso en cuestión involucra los tres elementos requeridos para la generación de desigualdad a partir de las actividades empresariales: corrupción, escalabilidad tecnológica y actividad monopólica. La historia, ahora investigada por la Cofece, se relaciona con la posible manipulación de la tasa de interés que sirve, entre otras cosas, para calcular el rendimiento de los bonos de deuda gubernamentales que el gobierno mexicano paga a los bancos e inversionistas que adquieren esos bonos, pagos que se hacen durante al menos el tiempo de vencimiento, si no, se renegocian.

En México, el valor del mercado de bonos gubernamentales, instrumentos que éste emite para financiar sus actividades cuando la recaudación puede no ser suficiente, alcanza los 6.2 billones de pesos anuales. Estos instrumentos pueden estar calculados a plazo fijo y se denominan, entre otros papeles, Cetes, Bonos M, Bondes D y Udibonos. El monto señalado puede alcanzar el $30 \%$ del PIB mexicano (Celis, 2019). Como se puede apreciar, el caso involucra la combinación de autoridades del sector financiero al más alto nivel y la participación de los niveles más altos de la jerarquía de bancos que operan en el mercado financiero, así como bancos de inversión en el extranjero.

El Banco de México (Banxico) es el encargado de organizar las subastas públicas donde los bonos de deuda emitidos por el gobierno mexicano serán adquiridos a cambio de una tasa de interés y un plazo de vencimiento determinado por inversionistas locales y extranjeros. Un ejemplo muy sencillo para explicar el funcionamiento de los bonos es el siguiente: un bono con valor de 100000 dólares a pagar en 20 años, con una tasa anual de 5\%, implicará que, al momento de vencimiento, en 20 años el gobierno habría pagado un interés equivalente al 100\% del monto del instrumento. Es decir, el inversionista obtiene una mayor ganancia si la tasa de interés y el plazo del documento son mayores.

Es conocido el efecto que tiene la tasa de interés en el funcionamiento de una economía moderna. Si ésta es alta, entonces la actividad económica puede ser menor debido a que es más conveniente invertir recursos en el sistema financiero, ya que la recompensa de decidir eso es mayor. Por otro lado, si la misma es baja, entonces el estímulo de invertir en ese sistema es menor y los inversionistas pueden optar por destinar sus recursos en la producción de bienes o servicios. La tasa de interés puede, según lo señalado, determinar el atractivo que tendrían los bonos que ofrece el gobierno en los mercados financieros.

En México, la instancia responsable de determinar la tasa de interés de referencia es Banxico, esto derivado de su autonomía en relación con la Secretaria de Hacienda. En teoría, el indicador del rendimiento de los bonos la calcula el banco usando criterios técnicos que promuevan la inversión 
en la economía mexicana y que la misma sirva para generar condiciones que fomenten el crecimiento. Asimismo, Banxico es responsable de determinar las acciones necesarias para contener la inflación en los términos que establezca la política económica del país.

A pesar de lo anterior, Banxico ha fijado una tasa de interés muy alta con relación a la inflación en el país. México tiene las tasas de interese más altas a corto y largo plazo, $8.45 \%$ y $7.68 \%$ respectivamente, entre 41 países, según la OCDE. Más aún, de acuerdo con el Banco de Pagos Internacionales (BIS, por sus siglas en inglés), el país tiene la tercera tasa de interés nominal, 8.25\%, más alta del mundo, sólo superado por Argentina, 63.7\%, y Turquía, con 19.7\%; sin embargo, si se toma en cuenta la tasa de inflación de esos dos países, se encuentra que las mismas alcanzan $55.8 \%$ y $19.5 \%$, respectivamente. En el caso mexicano, a julio del presente año, el mismo indicador alcanza 3.78\% (La Silla Rota, 2019).

En los casos de Argentina y Turquía se entiende que la tasa de interés sea más alta debido a la tasa de inflación que ellos tienen. El propósito de esos países es el de contener la inversión mediante la atracción de más capitales que salen de la economía real y van al mercado de capitales, de esa forma se busca desacelerar la actividad económica y que baje la inflación. Sin embargo, en el caso mexicano no se entiende que la relación tasa de interés/inflación sea 2.1, cuando en Argentina y Turquía, países que están en medio de graves crisis económicas, la relación sea 1.1 y 1 , respectivamente. La tasa de interés en México debiera fijarse en el rango de $4 \%$ a $4.5 \%$.

De acuerdo con los datos anteriores, cabe hacerse una pregunta importante para este artículo: ¿por qué Banxico fija una tasa de interés tan alta? Como se señaló anteriormente, la tasa de interés tiene relación con la actividad económica. Es decir, la misma se eleva cuando aumenta la inflación y las autoridades buscan ralentizar la economía y que se capten más capitales que vayan a los mercados financieros en lugar de la economía que produce bienes y servicios. El caso mexicano es atípico en ese sentido, ya que su economía no crece de forma importante y la inflación es baja, razones por las cuales se esperaría que la tasa bajara para provocar que la economía se reactive.

Otro dato que hace más extraño lo ocurrido con la tasa de interés en México es el flujo neto de inversión indirecta como efecto del lanzamiento de bonos de deuda gubernamental en el país. El resultado de ese flujo es negativo en 8.5 mil millones de dólares (La Silla Rota, 2019). Lo anterior significa que una alta tasa de interés no ha servido para conservar los capitales financieros en México, por lo que se puede detectar otra falla en la política de tasas de interés de Banxico. Aquí cabe señalar, entonces, que dicha política no cumple con los objetivos generalmente aceptados por los economistas sobre el rol que juegan las tasas de interés en la economía, no ha servido para hacer crecer la economía, contener la inflación y tampoco para conservar los capitales en el país. ¿A qué o quién ha servido elevar tanto las tasas de interés en México?

Uno de los usos que se puede dar a una elevada tasa de interés es el correspondiente al costo de la deuda pública del país. Es decir, los inversionistas que compran deuda mexicana tienen un rendimiento mayor en comparación con aquellos papeles de deuda que no tengan el mismo interés. Por ejemplo, Brasil, un país de desarrollo similar a México, paga $5 \%$ al año, según el BIS, contra $7.75 \%$ en México. Es decir, para un inversionista es más atractivo comprar deuda mexicana que brasileña. En cambio, el gobierno mexicano, o el contribuyente, paga más que el brasileño por la emisión de bonos por la misma cantidad de dinero. 
La mencionada investigación de la Cofece ofrece una explicación a lo señalado en el párrafo anterior: existe una colusión entre autoridades del Banxico, dirigido en ese entonces por Agustín Carstens, con los bancos que compraban los bonos en las subastas públicas que aquel organizaba cada mes. De esa forma, un banco compraba, con acuerdo de los demás, el $80 \%$ de los bonos y luego éste repartía a los demás los papeles de deuda negociados. Celis señala que los bancos involucrados en esa práctica son: BBVA-Bancomer, Santander, Citibanamex, JP Morgan, Bank of America, Barclays y Deutsche Bank (Celis, 2019).

De esa forma, el gobierno mexicano ha pagado durante casi 20 años millonarias cantidades de dinero producto de mantener la tasa de interés elevada para que los beneficios de esa manipulación del mercado de bonos sean repartidos entre los bancos y las autoridades del Banxico que se han beneficiado del uso discrecional de los recursos públicos. El banco central ha incumplido con su obligación con respecto a la determinación de la tasa de interés y su uso en la economía nacional. El contribuyente ha pagado por esa maniobra y las instituciones involucradas han actuado de forma extractiva. La cantidad de dinero involucrado en las subastas semanales organizadas por Banxico alcanzan los 50 mil millones de pesos (Celis, 2019).

El caso anterior muestra de qué forma las decisiones de un grupo de personas ubicadas en posiciones clave, autoridades y bancos, hacen pagar al contribuyente una mayor cantidad de dinero por la deuda pública de México a cambio de las comisiones y ganancias indebidas producto de la colusión de estas. La corrupción ha influido de forma fundamental en este ejemplo. También se ve que la escalabilidad del negocio financiero ha excluido a aquellos bancos y agentes que no cuentan con la capacidad para participar en las subastas públicas organizadas por Banxico. Esa exclusión permite, también, que se forme una competencia monopólica que termina por perjudicar el interés público.

\section{CONCLUSIONES}

La economía de mercado per se no crea la desigualdad económica. La libre empresa es un componente fundamental para el funcionamiento de este tipo de sistema económico. De hecho, habrá que añadir la racionalidad económica como un factor inseparable que condiciona a los individuos cuando deciden llevar a cabo actividades relacionadas con la creación y gestión de empresas.

La desigualdad económica, como consecuencia de la actividad empresarial, aumenta o disminuye por factores relacionados al entorno de las organizaciones y cómo, de acuerdo con los neoinstitucionalistas, las empresas se adaptan al entorno que enfrentan. En casos donde el entorno es favorable, aquellas tienden a ser más eficientes en sus operaciones debido a los menores costos que se relacionan con su operación. Por el contrario, en aquellos donde existan condiciones que favorezcan prácticas de corrupción, ventajas tecnológicas basadas no en la innovación sino en la apropiación de recursos y la prevalencia de monopolios, entonces las condiciones de competencia se distorsionan.

El costo de la señalada distorsión en el mercado, producto de los tres fenómenos estudiados en este artículo, son precursoras de la desigualdad económica que se ha convertido en un problema de dimensiones importantes. Por lo tanto, es pertinente señalar que la actividad empresarial en 
combinación con las condiciones del entorno puede generar prácticas rentistas y ofrecer beneficios inmerecidos que cimientan la desigualdad.

Las evidencias aquí mostradas pueden ser calificadas de reiterativas, más que novedosas, a la hora de puntualizar las implicaciones o consecuencias que tiene la desigualdad en una sociedad. Sin embargo, existe un aspecto que puede ser relevante y que merece ser explorado con mayor profundidad en futuras investigaciones: los factores o conductas precursoras de la desigualdad en las economías modernas tienen que ver más con acciones que cotidianamente llevan a cabo quienes dirigen empresas, bajo los contextos analizados en el presente trabajo, que con políticas públicas producto de la visión de burócratas que pueden encabezar diversas dependencias de gobierno.

Lo anterior nos revela que la desigualdad es causada por los ciudadanos y sus organizaciones y que ésta no es algo inherente o predeterminado de las sociedades a las que pertenecemos. Por lo tanto, debemos tener un papel más activo en el combate de las causas que la provocan, ya que, como se ha señalado, el costo es considerable y el precio más alto como producto de prácticas monopólicas que padecen y que finalmente terminan pagando los sectores más desfavorecidos de un país.

Las limitantes de espacio y tiempo que están implícitas en la elaboración de artículos como este, hacen que ciertos aspectos relevantes con el tema estudiado no hayan sido abordados con la extensión y detalle debidos. Un ejemplo de lo anterior lo representa la necesidad de ahondar en los mecanismos que han extendido las prácticas de corrupción y la obtención de ganancias inmerecidas producto de la colusión de un reducido número de actores económicos. Dicha colusión, producto de relaciones que no se explican como producto de mecanismos de oferta y demanda, tienden a perjudicar el interés de los consumidores en diversas formas.

\section{CONFLICTOS DE INTERÉS}

Los autores declaran que no presentan conflictos de interés financiero, profesional o personal que pueda influir de forma inapropiada en los resultados obtenidos o las interpretaciones propuestas.

\section{CONTRIBUCIÓN DE AUTORES}

Para el desarrollo de este proyecto todos los autores han realizado una contribución significativa especificada a continuación:

Juan Agustín Tapia-Alba: contribuyó con el desarrollo de la idea sobre la temática desarrollada, diseño, análisis, desarrollo y conclusión del trabajo de investigación.

Cesairé Chiatchoua: construcción metodológica de la idea desarrollada, diseño, análisis, desarrollo y conclusión del trabajo de investigación. 


\section{REFERENCIAS}

Acemoglu, D.; Robinson, J. A. (2012). Why Nations Fail? the origins of power, prosperity and poverty. Crown Publishers.

Albarrán, E. (2018). Corrupción le cuesta a México entre 5\% y 10\% del PIB: OCDE. El Economista. URL

Alpuche De La Cruz, E. (2017). La teoría de la innovación: un enfoque regional. En Chiatchoua, C y Tomta, D. (Coords.). Perspectivas de la innovación regional en México: propuesta de un modelo. (1st ed.). Colofon.

Banco Mundial. (2021). Índice de Gini - Ranking de países. Index Mundi. URL

Bapuji H. (2015). Individuals, interactions and institutions: How economic inequality affects organizations. Human Relations, v. 68, n. 7, 1059-1083. https://doi.org/10.1177/0018726715584804

Baumann, H. (2019). The corruption perception index and the political economy of governing at a distance. International Relations, v. 34, n. 4, 504-523. https://doi.org/10.1177/0047117819897312

Becerril, A. (2020, 16 de mayo). Thomas Piketty: la desigualdad en los tiempos del Covid-19. El Economista. $\underline{\mathrm{URL}}$

Bennett, C. J.; Zitikis, R. (2015). Ignorance, lotteries, and measures of economic inequality. The Journal of Economic Inequality, v. 13, n. 2, 309-316. https://doi.org/10.1007/s10888-015-9302-6

Celis, D. (2019, 14 de octubre). AMLO frente al lucrativo 'huachicoleo' financiero. El Financiero. URL

Comisión Federal de Competencia Económica. (2018). Poder de Mercado y Bienestar Social. URL

Conerly, B. (2018, 23 de septiembre). Business Is One Reason For Economic Inequality--And Also For Equality. Forbes. URL

Corporate Finance Institute. (s.f.). First Mover Advantage: The Advantage Gained by a Company that First Introduces a Product or Service to the Market. URL

Costas, A.; Germà, B. (2000). Privatización y posprivatización de servicios públicos: riesgos regulatorios e impuestos ocultos: el caso de España. CEPAL. https://repositorio.cepal.org/handle/11362/6879

Cuartas Ricaurte, J. (2016). ¿Desigualdad y pobreza como determinantes de la confianza generalizada? análisis con datos. Desarrollo y Sociedad, n. 76, 91-121. https://doi.org/10.13043/dys.76.2 
Dada Hirezi, H. (2015). Thomas Piketty y la desigualdad: una perspectiva desde El Salvador. Fundación Heinrich Böll. URL

Donadelli, M.; Fasan, M.; Magnanelli, B. S. (2014). The agency problem, financial performance and corruption: Country, industry and firm level perspectives. European Management Review, v. 11, n. 3-4, 259-272. https://doi.org/10.1111/emre.12038

Elliott, K. A. (2001). La corrupción en la economía global. Limusa.

Ensighten, F. (2016, 8 de junio). Innovation and Scalability Compete in Marketing Technology: BiModal Framework Supports Both. URL

Everhart, S. S.; Martinez-Vazquez, J.; McNab, R. M. (2009). Corruption, Governance, Investment and Growth in Emerging Markets. Applied Economics, v. 41, n. 13, 1579-1594. https://doi.org/10.1080/00036840701439363

Falcone-Treviño, G. F.; Jiménez-Galán, J. L.; Tinajero Mallozzi, Z. L.; Serna-Hinojosa, J. A. (2018). Gestión de Innovación en Negocios. VinculaTégica EFAN, v. 3, n. 3. 463-478. URL

Flores Ramírez, G. (2018, 16 de enero). TLCAN, noticias falsas y la megamulta. El Economista. URL

Flores, E. (2018, 5 de noviembre). Siete corporativos que imponen precios al país, pero que para el Gobierno «no son monopolios». SinEmbargo. $\underline{\text { URL }}$

Glennie, A.; Gabriel, M. (2017, 11 de septiembre). Is there a role for innovation in tackling global inequality? $\underline{\mathrm{URL}}$

Goldberg, G. S. (2012). Economic Inequality and Economic Crisis: A Challenge for Social Workers. Social Work, v. 57, n, 3, 211-224. https://doi.org/10.1093/sw/sws005

Graham, V. (2018, 26 de octubre). Tech Giants are Bigger Antitrust Threats than Standard Oil: Stiglitz. Bloomberg Law. URL

Hoffman, F. (2019, 24 de enero). Business Model Scalability: Internal vs External. URL

Houle, C. (2018). Does economic inequality breed political inequality? Democratization, v. 25, n. 8 , 1500-1518. https://doi.org/10.1080/13510347.2018.1487405

Instituto Internacional de Gobernabilidad Barcelona. (1998). Douglass C. North: la teoría económica neo-institucionalista y el desarrollo latinoamericano. $\underline{\mathrm{URL}}$

Johnson, M. (2001). Funcionarios públicos, intereses privados y democracia sustentable: cuando la política y la corrupción se juntan. En Elliott, K. A (Ed.). La corrupción en la economía global (83108). Limusa. 
Keefer, P.; Knack, S. (2007). Boondoggles, Rent-Seeking, and Political Checks and Balances: Public Investment under Unaccountable Governments. Review of Economics and Statistics, v. 89, n. 3, 566-572. https://doi.org/10.1162/rest.89.3.566

Krugman, P. R.; Obstfeld, M. (2006). Economía Internacional Teoría y Política (7 Ed.). Pearson Educación.

Lara, F. (2018, 6 de junio). Costo de la corrupción en México equivale a 18\% del PIB. Milenio. URL

Lin, B. C. A. (2007). A new vision of the knowledge economy. Journal of Economic Surveys, v. 21, n. 3, 553-584. https://doi.org/10.1111/j.1467-6419.2007.00514.x

Mauro, P. (2001). Los efectos de la corrupción sobre el crecimiento, la inversión y el gasto público: análisis comparativo de varios países. En Elliott, K. A (Ed.). La corrupción en la economía global (109-136). Limusa.

Medá, P. (2016, 16 de septiembre). How Business Scalability Works. URL

Moser, D. J. (2018). The Platform Economy: Strategies, Governance, and Business Models. [Doctoral Dissertation, Universität St. Gallen].

Neme-Castillo, O.; Valderrama-Santibáñez, A. L.; Chiatchoua, C. (2021). Análisis de las competencias núcleo y el desarrollo empresarial en las MIPES: estudio de caso. Revista CEA, v. 7, n. 13, e-1599. https://doi.org/10.22430/24223182.1599

North, D. C. (1993). Instituciones, cambio institucional y desempeño económico. Fondo de Cultura Económica.

Organización de las Naciones Unidas para la Educación, la Ciencia y la Cultura. (s.f.). How much does your country invest in R\&D? UNESCO Institute for Statistics. $\underline{\text { URL }}$

Organización para la Cooperación y el Desarrollo Económico. (2015). Consequences of Corruption at the Sector Level and Implications for Economic Growth and Development. OCDE.

Organización para la Cooperación y el Desarrollo Económico. (2017). Estudios Económicos de la OCDE México 2017. OCDE. URL

Organización para la Cooperación y el Desarrollo Económico. (2018). Going for Growth 2018. URL

Ortiz Baquero, I. S.; Solano Osorio, D. A. (2016). La libre competencia económica y la protección del medio ambiente: una aproximación al estudio de los acuerdos voluntarios de cumplimiento ambiental. Revista E-mercatoria, v. 15, n. 1. URL

Reyes Hernández, M. (2018). Corrupción y Desarrollo ¿realmente la primera afecta al segundo? URL 
Silla Rota. (2019, 12 de agosto). Banxico, en top 3 de las tasas de interés más altas del mundo, pero vuelan capitales. $\underline{\mathrm{URL}}$

SinEmbargo. (2018, 12 de octubre). Monopolios causan sobreprecios de hasta 98\% en tortillas, frutas, lácteos, verduras, medicinas. $\underline{\mathrm{URL}}$

Stiglitz, J. (2015). El Precio de la Desigualdad: El 1\% de población tiene lo que el 99\% necesita. Taurus.

Toro-García, A. F.; Gutiérrez-Vargas, C. C.; Correa-Ortiz, L. C. (2020). Estrategia de gobierno digital para la construcción de Estados más transparentes y proactivos. Trilogía Ciencia Tecnología Sociedad, v. 12, n. 22, 71-102. https://doi.org/10.22430/21457778.1235

Vail, M. I. (2009). Left of Eden: The changing politics of economic inequality in contemporary Germany. German Politics, v. 18, n. 4, 559-576. https://doi.org/10.1080/09644000903349408

Worldometers. (2019, 8 de septiembre). Global GDP This Year. URL 